\title{
Weak deflection angle of Kazakov-Solodukhin black hole in plasma medium
}

\author{
Wajiha Javed, ${ }^{1, *}$ Iqra Hussain, ${ }^{1, \dagger}$ and Ali Övgün ${ }^{2, \ddagger}$ \\ ${ }^{I}$ Division of Science and Technology, University of Education, Township-Lahore, Pakistan \\ ${ }^{2}$ Physics Department, Eastern Mediterranean University, \\ Famagusta, 99628 North Cyprus via Mersin 10, Turkey.
}

(Dated: July 3, 2021)

\begin{abstract}
In this paper, we study light rays in a Kazakov-Solodukhin black hole. To this end, we use the optical geometry of the Kazakov-Solodukhin black hole within the Gauss-bonnet theorem. We first show the effect of the deformation parameter $a$ on the Gaussian optical curvature, and then we use the modern method popularized by Gibbons and Werner to calculate the weak deflection angle of light. Our calculations of deflection angle show how gravitational lensing is affected by the deformation parameter $a$. Moreover, we demonstrate the effect of a plasma medium on weak gravitational lensing by the Kazakov-Solodukhin black hole. We discuss that the increasing the deformation parameter $a$, will increase the weak deflection angle of the black hole. Our analysis also uncloak how one may find a observational evidence for a deformation parameter on the deflection angle.

PACS numbers: 95.30.Sf, 98.62.Sb, 97.60.Lf

Keywords: Relativity; Gravitation Lensing; Kazakov-Solodukhin black hole; Gauss-Bonnet Theorem; Deflection angle; Plasma medium
\end{abstract}

\section{INTRODUCTION}

In 1915, Einstein cleverly came up with a new idea of theory of general relativity (GR) [1], that has been undergone through a number of experiments [2-4]. It was founded that the experimental consequences and the conceptual predictions both were well fitted with each other. One of the greatest challenges in theoretical physics is the unification of quantum field theory and Einstein's general relativity into a theory of quantum gravity. Using the known physical laws, the theories are not compatible with each other. However, to understand the inside of the Black hole and the Big Bang, these theories should be combined and the quantum corrections must be located in the Schwarzcshild solution according to the various approaches to unify them. Kazakov and Solodukhin show that the generalization of the Schwarzschild solution is possible by neglecting the non-spherical deformations using the effective scalar-tensor gravity [5] and its properties are studied in [6-8]. Furthermore gravitational lensing (GL) is one of the famous prediction and numerous individuals also verified these experiments [9, 10]. In 1801, GL was initially suggested by Soldner [11]. Gravitational lensing is a powerful tool to understand not only dark and massive objects [12-14] but it is also very effective to study black holes (BHs) and wormholes [15-33].

A BH is an astronomical object having such strong gravity that nothing could escape from it even electromagnetic radiations such as light [34]. The boundary of a BH from which nothing could escape is called event horizon. Black hole behaves as an ideal black body because it reflects no light $[35,36]$. According to GR, the light beam is deflected by a small angle if it passes closely to mass $M$ with a huge effect parameter $b$ defined as [37, 38]:

$$
\gamma=\frac{2 R_{S}}{b}=\frac{4 M}{b}, G=c=1 .
$$

If $b \leq R_{S}$ then the deflection will be strong, where $R_{S}=2 M$ represents the Schwarzschild radius of the floating body. Another name for deflection angle is "Einstein angle".

In 2008, Gibbons and Werner (GW) founded another geometrical technique to process the deflection angle of light by the implementation of Gauss-Bonnet theorem (GBT) to the optical geometry for asymptotically flat static BHs [39]. Gibbons and Werner proclaimed that this formula gives the perfect result for Schwarzschild BH [39] in weak fields. Afterwards, this formula was enlarged to find the deflection angle of Kerr BH [40] and other objects such as rotating global monopole, cosmic string, wormholes and other solutions [41-91]. Currently, GW have changed the common point of view associated to the technique of calculating the deflection angle. By using GBT, one can obtain a beautiful expression to find the deflection angle. Now, let shortly discuss the GBT also known as Gibbons-Werner method that tie up the topologically surfaces and show that the deflection

\footnotetext{
*Electronic address: wajiha.javed@ue.edu.pk; wajihajaved84@yahoo.com

†Electronic address: iqrahussain057@gmail.com

${ }^{\ddagger}$ Electronic address: ali.ovgun@emu.edu.tr
} 
angle can be calculated as follows:

$$
\beta=-\int_{0}^{\pi} \int_{\frac{b}{r \sin \phi}}^{\infty} \mathcal{K} d S
$$

where the $\mathcal{K}$ is the Gaussian optical curvature. The above equation is valid for the deflection angle of asymptotically flat spacetime, if we have non asymptotically flat metric then, one should use the finite distance corrections [43]. Ishihara et al. calculated the deflection angle of light for the finite distance (huge impact parameter) using the GBT when the source and the observer are at asymptotically flat region [43]. Then, Crisnejo and Gallo [42] use the GBT to obtain the gravitational deflections of light in a plasma medium in a black hole spacetime.

The aim of this work is to calculate the deflection angle of the Kazakov-Solodukhin black hole [5-7] by using the GBT. After that, we will contemplate the impact of plasma medium on the desired black hole and discuss the effect of the deformation parameter $a$ on the deflection angle in vacuum and in plasma medium.

The work is formed as follows. In section 2, we first review the Kazakov-Solodukhin black hole. Then we calculate its optical geometry and the Gaussian optical curvature. In section 3, we devote this section to find deflection angle of the KazakovSolodukhin black hole in weak field limits. In section 4, we calculate the deflection angle in plasma medium. Furthermore, we determine the graphical behavior of the deflection angle of the Kazakov-Solodukhin black hole. We conclude in section 5.

\section{KAZAKOV-SOLODUKHIN BLACK HOLE}

The Kazakov-Solodukhin bh spacetime is described by the following line-element [5]

$$
d s^{2}=-B(r) d t^{2}+\frac{d r^{2}}{B(r)}+r^{2}\left(d \theta^{2}+\sin ^{2} \theta d \phi^{2}\right)
$$

with the metric function

$$
B(r)=\left(\frac{\sqrt{r^{2}-a^{2}}}{r}-\frac{2 M}{r}\right)
$$

and the asymptotic expression for the metric $r>>a$ is

$$
B(r) \approx 1-\frac{a^{2}}{2 r^{2}}-\frac{2 M}{r}
$$

where $\mathrm{BH}$ mass is indicated by $M$ and $a$ is the deformation parameter.

For static and spherically symmetric metric, the light source and the observer are taken in the equatorial plane as $\left(\theta=\frac{\pi}{2}\right)$. As we are working in null geodesics so, put $d s^{2}=0$ and we obtain the corresponding optical metric

$$
d t^{2}=\frac{d r^{2}}{\left(1-\frac{a^{2}}{2 r^{2}}-\frac{2 M}{r}\right)^{2}}+\frac{r^{2} d \phi^{2}}{1-\frac{a^{2}}{2 r^{2}}-\frac{2 M}{r}} .
$$

The Gaussian optical curvature $\mathcal{K}$ can also be rewritten in:

$$
\mathcal{K}=\frac{R_{r \phi r \phi}}{2}
$$

which yields for the optical metric of Kazakov-Solodukhin bh as:

$$
\mathcal{K} \approx-\frac{2 M}{r^{3}}-\frac{3 a^{2}}{2 r^{4}}-\frac{M^{2}}{r^{5}}+\frac{3 M a^{2}}{r^{5}}-\frac{a^{4}}{4 r^{6}} .
$$

After simplifying, we can write it in weak field limits as follows:

$$
\mathcal{K} \approx-\frac{2 M}{r^{3}}-\frac{3 a^{2}}{2 r^{4}}+\frac{3 M a^{2}}{r^{5}}+\mathcal{O}\left(M^{2}, a^{4}\right) .
$$




\section{DEFLECTION ANGLE OF KAZAKOV-SOLODUKHIN BH}

In this section, we calculate the deflection angle of a Kazakov-Solodukhin bh using the GBT which provides relation between the intrinsic geometry of the spacetime and its topology of the region $\mathcal{A}_{R}$ with boundary $\partial A_{R}$, stated as [39]

$$
\iint_{\mathcal{A}_{R}} \mathcal{K} d S+\oint_{\partial \mathcal{A}_{R}} k d t+\sum_{j} \epsilon_{j}=2 \pi \mathcal{X}\left(\mathcal{A}_{R}\right)
$$

where $\mathcal{K}$ is the Gaussian curvature and $k$ is the geodesic curvature, stated as $k=\bar{h}\left(\nabla_{\dot{\gamma}} \dot{\gamma}, \ddot{\gamma}\right)$ such a way that $\bar{h}(\dot{\gamma}, \dot{\gamma})=1$, here $\ddot{\gamma}$ represents unit acceleration vector and the $\epsilon_{j}$ is the exterior angle at the jth vertex. As $R \rightarrow \infty$, our two jump angles become $\pi / 2$ or we can say that the sum of the two jump angles to the observer and source satisfies $\theta_{O}+\theta_{S} \rightarrow \pi$. The Euler characteristic is $\mathcal{X}\left(\mathcal{A}_{R}\right)=1$, as $\mathcal{A}_{R}$ is non singular. Consequently, we rewrite it as,

$$
\iint_{\mathcal{A}_{R}} \mathcal{K} d S+\oint_{\partial \mathcal{A}_{R}} k d t+\epsilon_{j}=2 \pi \mathcal{X}\left(\mathcal{A}_{R}\right)
$$

here, $\epsilon_{j}=\pi$ demonstrates that $\gamma_{\bar{h}}$ also the entire jump angle is a geodesic. Now, geodesic curvature $k\left(C_{R}\right)=\left|\nabla_{\dot{C}_{R}} \dot{C}_{R}\right|$ should be calculated. Since, we can evaluate the geodesic curvature's radial part:

$$
\left(\nabla_{\dot{C}_{R}} \dot{C}_{R}\right)^{r}=\dot{C}_{R}^{\phi} \partial_{\phi} \dot{C}_{R}^{r}+\Gamma_{\phi \phi}^{r}\left(\dot{C}_{R}^{\phi}\right)^{2}
$$

For large $R, C_{R}:=r(\phi)=r=$ const. The christofell symbols that are related to the optical geometry are given in the last equation $\Gamma_{\phi \phi}^{r}$. It is obvious from the above equation that initial term will vanish as topological effect is not involved and the second term will be calculated by using $k\left(C_{R}\right)=\left|\nabla_{\dot{C}_{R}} \dot{C}_{R}\right|$. The geodesic curvature is obtained as follows:

$$
\left(\nabla_{\dot{C}_{R}^{r}} \dot{C}_{R}^{r}\right)^{r} \rightarrow \frac{1}{R}
$$

Subsequently, we came up $k\left(C_{R}\right) d t=d \phi$. Then the GBT becomes

$$
\iint_{\mathcal{A}_{R}} \mathcal{K} d s+\oint_{\partial \mathcal{A}_{R}} k d t={ }^{R \rightarrow \infty} \iint_{S_{\infty}} \mathcal{K} d S+\int_{0}^{\pi+\beta} d \phi .
$$

For calculating the deflection angle in the weak deflection limit at 0th order, we use the light beam which follows a straight line approximation and is defined as $r(t)=b / \sin \phi$. The deflection angle is then obtained by using

$$
\beta=-\int_{0}^{\pi} \int_{b / \sin \phi}^{\infty} \mathcal{K} \sqrt{\operatorname{detg}} d r d \phi
$$

where

$$
\mathcal{K} \sqrt{\operatorname{det} g}=\left(\frac{-15 M^{2} a^{2}}{2 r^{5}}-3 \frac{M^{2}}{r^{3}}-3 \frac{a^{2} M}{r^{4}}-2 \frac{M}{r^{2}}-\frac{3 a^{2}}{2 r^{3}}\right) .
$$

After using Eq. (15) into Eq. (14), the deflection angle thus becomes

$$
\beta \approx \frac{4 M}{b}+\frac{3 a^{2} \pi}{8 b^{2}}+\frac{4 a^{2} M}{3 b^{3}}+\mathcal{O}\left(M^{2}, a^{4}\right)
$$

Thus, one can immediately observe how the deformation parameter $a$ affect the weak deflection angle of the KazakovSolodukhin bh.

\section{GRAPHICAL ANALYSIS FOR NON-PLASMA MEDIUM}

In this section, we study the graphical analysis of deflection angle on Kazakov-Solodukhin bh. To check the effect of deformation parameter $a$ and impact parameter $b$ on the deflection angle we likewise illustrate the deflection angle on plots. 
A. Deflection angle $\beta$ w.r.t deformation parameter $a$
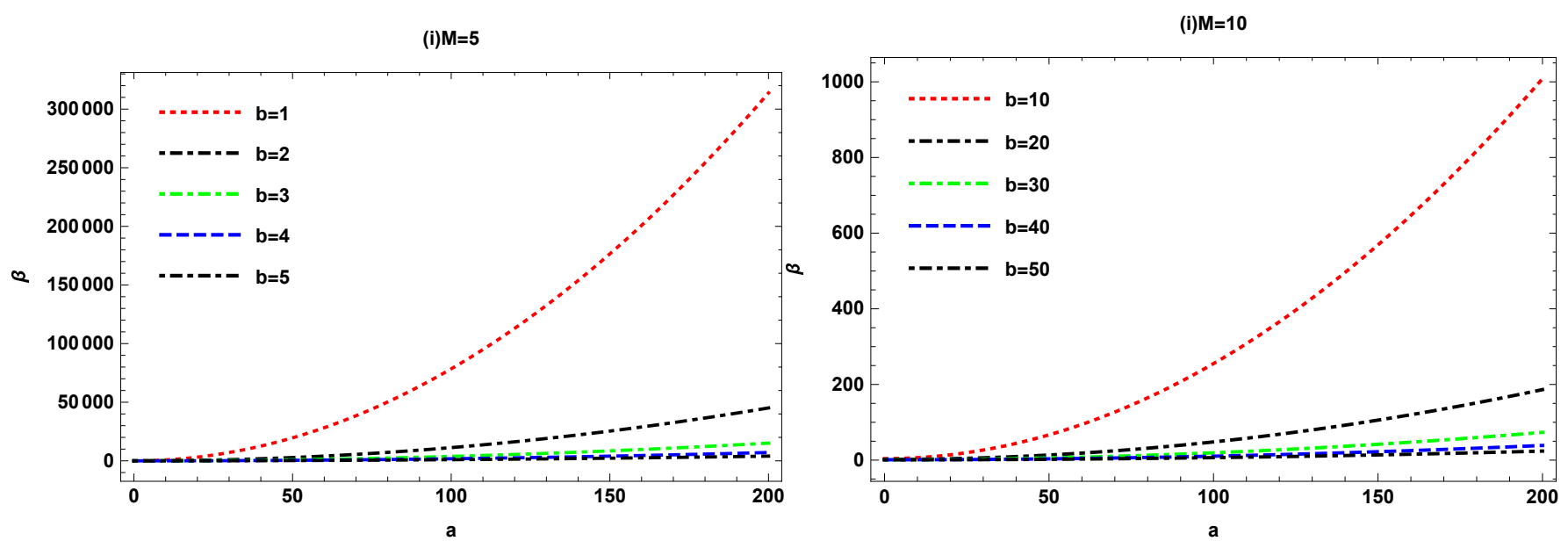

Figure 1: Relation between $\beta$ and $a$.

- Figure 1 This plot shows the behavior of $\beta$ w.r.t $a$ for different values of $b$ and by selecting fix value of $M$.

1. The left plot shows that the deflection angle is decreasing for increasing the impact parameter and deflection angle promptly decreases in between $1<b<2$.

2. We show that in the right plot the deflection angle is decreasing for the increasing of $b$ fo the large values of $b$.

\section{B. Deflection angle $\beta$ w.r.t Impact parameter $b$}

(i) $a=10$

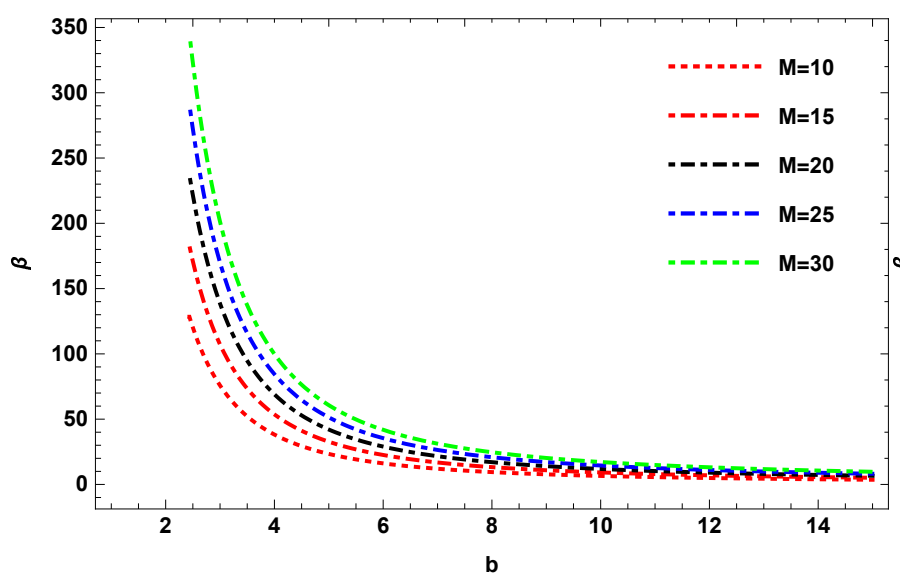

(i) $a=5$

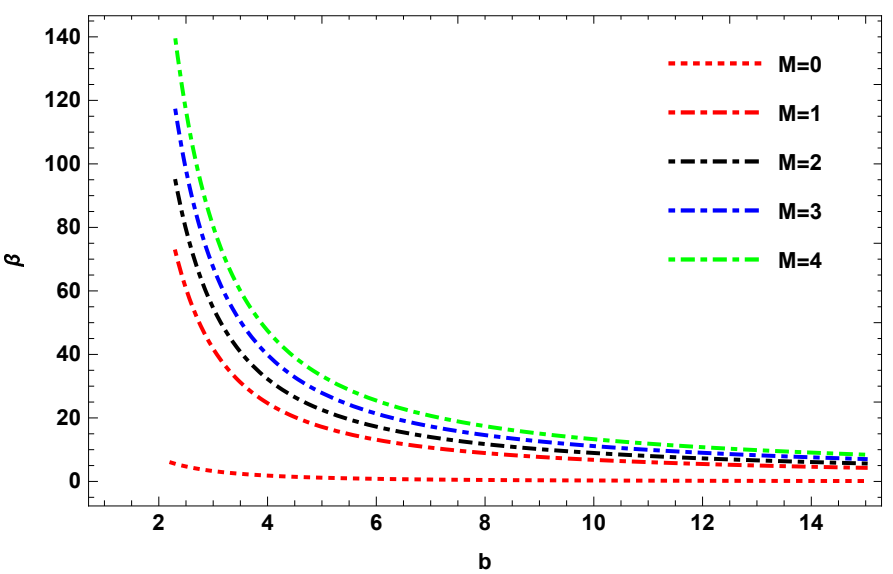

Figure 2: Relation between $\beta$ and $b$.

- Figure 2 shows the behavior of $\beta$ w.r.t $b$ by fixing the value of $a$ and varying $M$.

1. In the left plot, it can be noticed that the deflection angle $\beta$ is gradually increasing for the large values of $M$.

2. In the right plot, it can be observed that the deflection angle $\beta$ gradually increase for small variation of $M$.

Therefore, it can be concluded that the deflection angle increases in both the cases (for large and small values of $M$ ). 

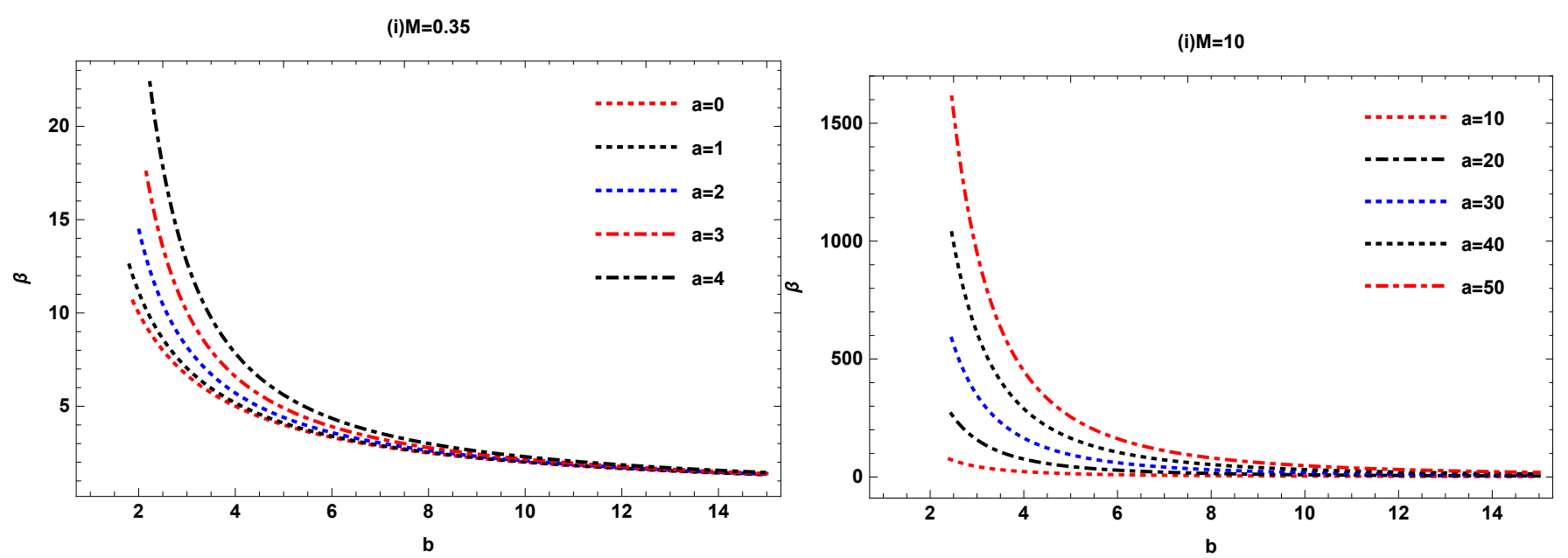

Figure 3: Relation between $\beta$ and $b$.

- Figure 3 shows the behaviour of deflection angle w.r.t impact parameter by keeping the value of $M$ fixed and giving variation to the value of deformation parameter $a$.

1. In both the left and the right plots, it can be noticed that $\beta$ gradually increases in both the cases (for small and large variation of $a$ ).

\section{Deflection angle $\beta$ w.r.t $M$}

(i) $a=5$

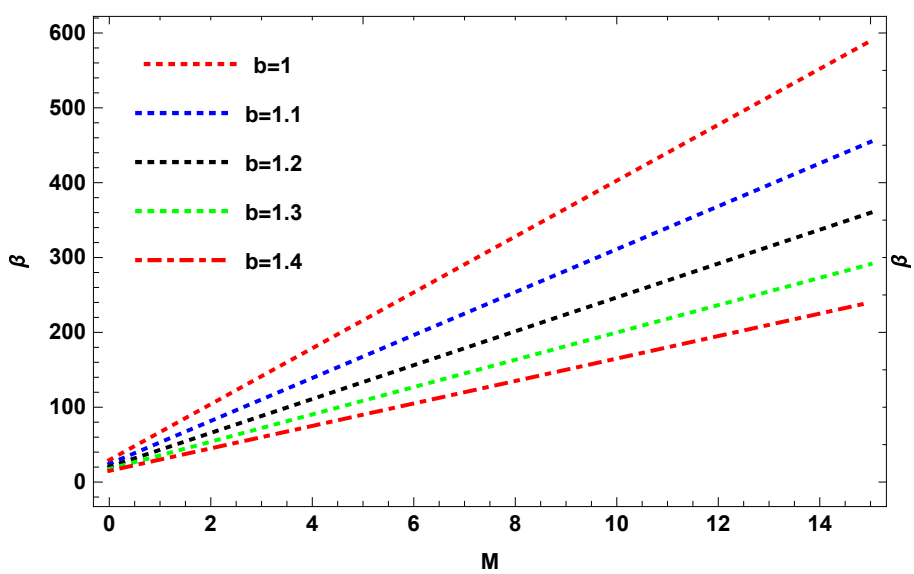

Figure 4: Relation between $\beta$ and $M$. (i) $b=5$

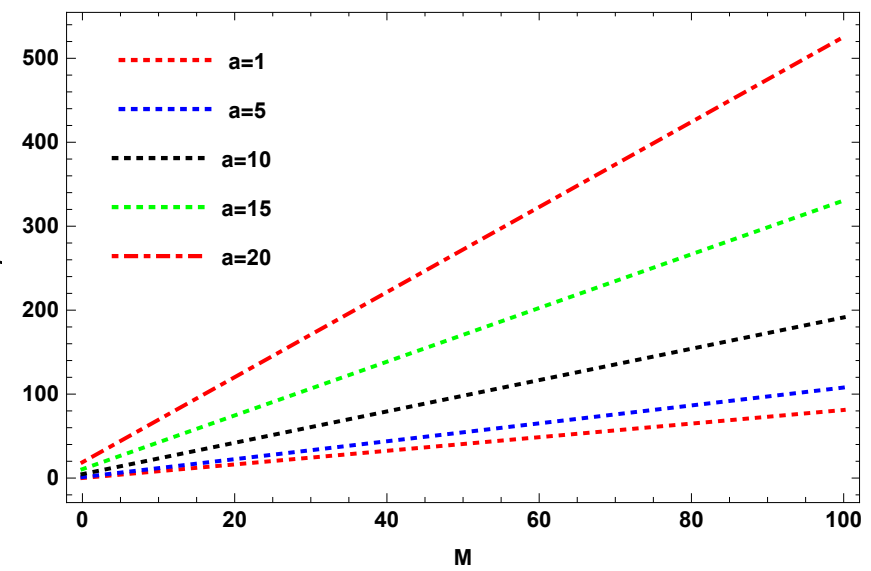

M

- Figure 4 shows the behavior of $\beta$ w.r.t $M$ by fixing $a$ and for small variation of $b$ and for fixed value of $b$ and for large values of $a$ respectively.

1. In the left plot, we noticed $\beta$ is decreases for the small variations of $b$.

2. In the right plot, we noticed $\beta$ is increases for large variations of $a$.

\section{GRAVITATIONAL LENSING BY KAZAKOV-SOLODUKHIN BH IN PLASMA MEDIUM}

In this section, we study the effect of plasma medium on the gravitational lensing of a Kazakov-Solodukhin bh. For this purpose, we consider the corresponding $\mathrm{BH}$ in plasma having refractive index $n$,

$$
n^{2}(r, \omega(r))=1-\frac{\omega_{e}^{2}(r)}{\omega_{\infty}^{2}(r)} .
$$


The refractive index reads

$$
n(r)=\sqrt{1-\frac{\omega_{e}^{2}}{\omega_{\infty}^{2}}\left(1-\frac{a^{2}}{2 r^{2}}-\frac{2 M}{r}\right)}
$$

Similarly as before, we obtain the corresponding optical metric as follows:

$$
d t^{2}=g_{x y}^{o p t} d x^{x} d x^{y}=n^{2}\left[\frac{d r^{2}}{B^{2}(r)}+\frac{r^{2} d \vartheta^{2}}{B(r)}\right]
$$

the determinant $\left(g_{x y}^{o p t}\right)$ of the above optical metric is defined as follows:

$$
\sqrt{g^{\text {opt } t_{x y}}}=r\left(1-\frac{\omega_{e}^{2}}{\omega_{\infty}^{2}}\right)+M\left(3-\frac{\omega_{e}^{2}}{\omega_{\infty}^{2}}\right)+\frac{a^{2}}{4 r}\left(3-\frac{\omega_{e}^{2}}{\omega_{\infty}^{2}}\right) .
$$

By using Eq. (19), we can find the following three non-zero christofell symbols as

$$
\begin{gathered}
\Gamma_{00}^{0}=\left(1+\frac{\omega_{e}^{2} B}{\omega_{\infty}^{2}}\right)\left[-B^{\prime} B^{-1}\left(1-\frac{\omega_{e}^{2} B}{\omega_{\infty}^{2}}\right)-\frac{B^{\prime} \omega_{e}^{2}}{2 \omega_{\infty}^{2}}\right], \\
\Gamma_{10}^{1}=\left(1+\frac{\omega_{e}^{2} B}{\omega_{\infty}^{2}}\right)\left[r^{-1}\left(1-\frac{\omega_{e}^{2} B}{\omega_{\infty}^{2}}-\frac{B^{\prime} B^{-1}}{2}\left(1-\frac{\omega_{e}^{2} B}{\omega_{\infty}^{2}}\right)-\frac{B^{\prime} \omega_{e}^{2}}{2 \omega_{\infty}^{2}}\right]\right.
\end{gathered}
$$

and

$$
\Gamma_{11}^{0}=\left(1+\frac{B \omega_{e}^{2}}{\omega_{\infty}^{2}}\right)\left[-r B\left(1-\frac{B \omega_{e}^{2}}{\omega_{\infty}^{2}}\right)+\frac{r^{2} B^{\prime}}{2}\left(1-\frac{B \omega_{e}^{2}}{\omega_{\infty}^{2}}\right)+\frac{r^{2} B}{2} \frac{B^{\prime} \omega_{e}^{2}}{\omega_{\infty}^{2}}\right] .
$$

Gaussian optical curvature in terms of curvature tensor can be stated as

$$
\mathcal{K}=\frac{R_{r \vartheta r \vartheta}\left(g^{o p t_{x y}}\right)}{\operatorname{det}\left(g^{o p t_{x y}}\right)},
$$

by the use of Eq. (21) in the weak field limit Gaussian optical curvature is written as:

$$
\mathcal{K} \approx 12 \frac{M^{2} \omega_{e}^{2}}{\omega_{\infty}^{2} r^{4}}+3 \frac{M^{2}}{r^{4}}-3 \frac{M \omega_{e}^{2}}{\omega_{\infty}^{2} r^{3}}-2 \frac{M}{r^{3}}-16 \frac{M^{2} a^{2} \omega_{e}^{2}}{\omega_{\infty}^{2} r^{6}}+13 \frac{M \omega_{e}^{2} a^{2}}{\omega_{\infty}^{2} r^{5}}+3 \frac{M a^{2}}{r^{5}}-\frac{5 a^{2} \omega_{e}^{2}}{2 \omega_{\infty}^{2} r^{4}}-\frac{3 a^{2}}{2 r^{4}}
$$

For calculating angle in the weak field limits of the light rays, we follow a straight line approximation so that we can utilize the condition $r=\frac{b}{\sin \phi}$ within the GBT

$$
\beta=-\lim _{R \rightarrow 0} \int_{0}^{\pi} \int_{\frac{b}{\sin \vartheta}}^{R} \mathcal{K} d S
$$

By using Eq. (22) within the Eq. (23), the deflection angle $\beta$ in plasma medium is found as follows:

$$
\beta \approx \frac{4 M}{b}+\left(\frac{3 \pi}{8 b^{2}}+\frac{4 M}{3 b^{3}}\right) a^{2}+\left(\frac{5 M}{b \omega_{\infty}^{2}}+\left(\frac{17 \pi}{32 b^{2} \omega_{\infty}^{2}}-\frac{4 M}{3 b^{3} \omega_{\infty}^{2}}\right) a^{2}\right) \omega_{e}^{2}
$$

It is shown that the photon rays move in a medium of homogeneous plasma and taking $\left(\frac{\omega_{e}}{\omega_{\infty}} \rightarrow 0\right)$, Eq. (24) reduces to Eq. (16), and the effect of the plasma is removed.

\section{GRAPHICAL ANALYSIS FOR PLASMA MEDIUM}

In this section, we study the graphical behavior of deflection angle $\beta$ in the plasma medium. Moreover, we also overview the physical significance of these plots to investigate the impact of plasma medium as well as the deformation parameter $a$. Here, we consider $M=1, \frac{\omega_{e}}{\omega_{\infty}}=10^{-1}$ and give variation to the impact parameter $b$ and deformation parameter $a$ in order to acquire these plots. 
A. Deflection angle $\beta$ w.r.t $a$

(i) $b=5$

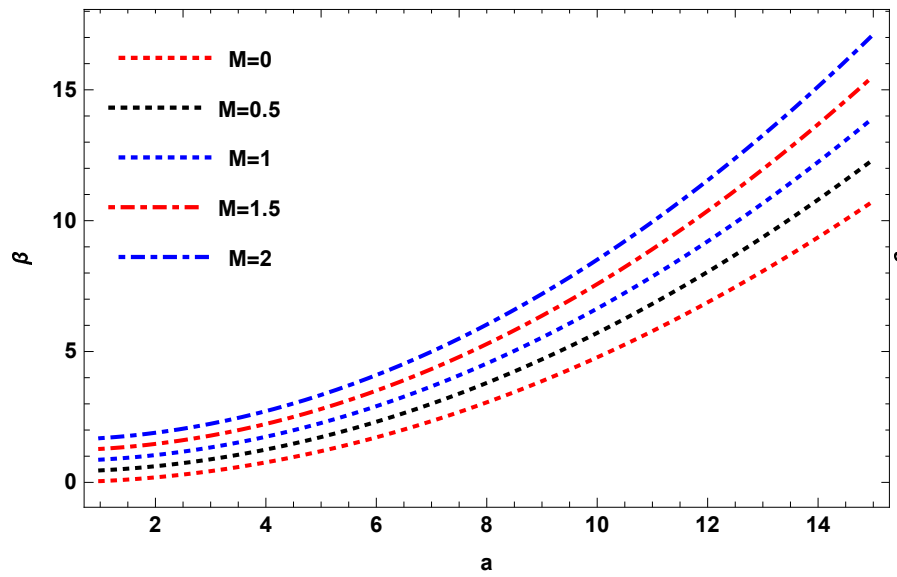

(i) $M=1$

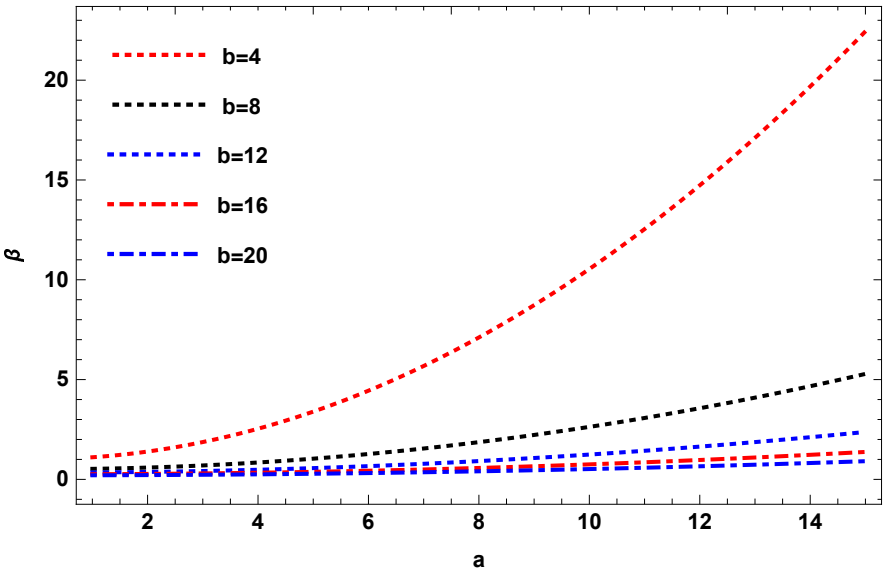

Figure 5: Relation between $\beta$ and $a$.

- Figure 5 displays the behavior of $\beta$ w.r.t $a$ for fix values of $b$ and for varying the values of $M$ and for fix values of $M$ and for varying the values of $b$ respectively.

1. In the left plot, we observe that the deflection angle gradually increases for small variation of $M$ and then eventually, goes to positive infinity.

2. In the right plot, we observed that deflection angle exponentially decreases for large variation of impact parameter and deflection angle rapidly decrease for $4<b<8$.

\section{B. Deflection angle $\beta$ w.r.t $b$}

(i) $a=5$

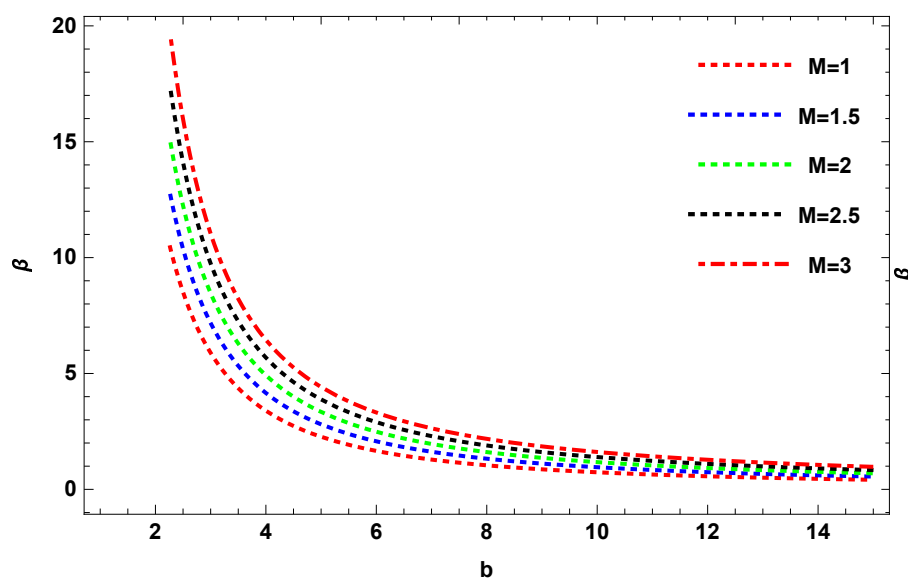

(i) $M=10$

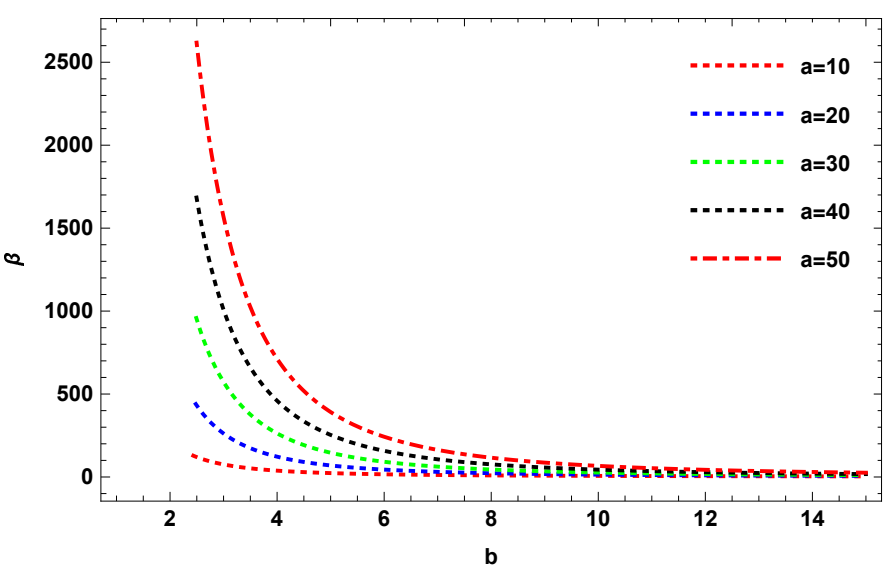

Figure 6: Relation between $\beta$ and $b$.

- Figure 6 shows the behavior of $\beta$ w.r.t $b$ for fix values of $a$ and for varying the values of $M$ and for fix values of $M$ and for varying the values of $a$ respectively.

1. In the left plot, we observed that deflection angle $\beta$ is gradually increases for small variation of $M$ and approaches to positive infinity.

2. In the right plot, we observed that deflection angle $\beta$ is gradually increases for huge variation of $a$ and approaches to positive infinity. 


\section{Deflection angle $\beta$ w.r.t $M$}
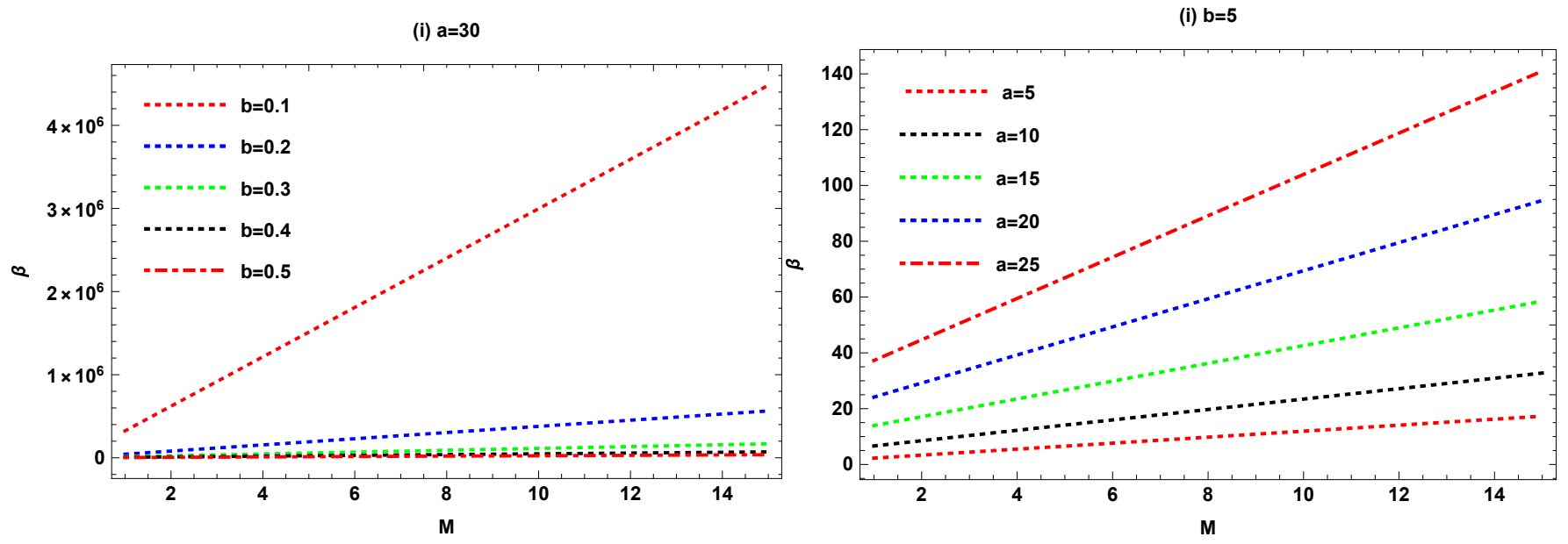

Figure 7: Relation between $\beta$ and $M$.

- Figure 7 demonstrate the behavior of deflection angle $\beta$ w.r.t $M$ by fixing the values of deformation parameter $a$ and by varying the values of impact parameter $b$ and fixing the values of deformation parameter $b$ and by varying the values of impact parameter $a$ respectively

1. In the left plot, we observed that deflection angle $\beta$ exponentially decreases for small variations of impact parameter $b$ and deflection angle $\beta$ rapidly decrease for $0.1<b<0.2$.

2. In the right plot, we observed that deflection angle gradually increases for large variations of deformation parameter $a$.

\section{SUMMARY}

In this paper, we have obtained the deflection angle for Kazakov-Solodukhin bh in both plasma and non-plasma medium. First, we have used a method proposed by GW known as GBT (a new geometric technique), to calculate the deflection angle for the desired $\mathrm{BH}$. We have obtained the deflection angle $\beta$ of light by integrating a domain area outside the impact parameter $b$, which shows that gravitational lensing is a global effect and is a good tool to investigate the features of the $\mathrm{BH}$. In our analysis, we have shown the effect of the deformation parameter $a$ on the deflection angle $\beta$ in the weak field limit. Hence, the computed deflection angle (16) is as follow

$$
\beta \approx \frac{4 M}{b}+\frac{3 a^{2} \pi}{8 b^{2}}+\frac{4 a^{2} M}{3 b^{3}}+\mathcal{O}\left(M^{2}, a^{4}\right)
$$

It is noted that if we select $a=0$ in the above equation, the calculated deflection angle reduces to the Schwarzschild deflection angle up to the first order term. Furthermore, we likewise study the graphical analysis of deflection angle for the KazakovSolodukhin bh. Moreover, by considering the homogeneous plasma medium, we have shown that the deflection angle of light is (24):

$$
\beta_{\text {plasma }} \approx \frac{4 M}{b}+\left(\frac{3 \pi}{8 b^{2}}+\frac{4 M}{3 b^{3}}\right) a^{2}+\left(\frac{5 M}{b \omega_{\infty}^{2}}+\left(\frac{17 \pi}{32 b^{2} \omega_{\infty}^{2}}-\frac{4 M}{3 b^{3} \omega_{\infty}^{2}}\right) a^{2}\right) \omega_{e}^{2}
$$

Now, in order to remove the plasma effect, we neglect this term $\left(\frac{\omega_{e}}{\omega_{\infty}} \rightarrow 0\right)$ and observe that the deflection angle in plasma medium reduces into vacuum results.

We have also studied the graphical analysis of deflection angle w.r.t impact parameter $\mathrm{b}$, deformation parameter $a$. The outcomes achieved from the influence of deflection angle stated in the paper are summed up as follows:

Deflection angle w.r.t Impact parameter:

1. Our main aim is to discuss the behavior of deflection angle for different values of impact parameter, deformation parameter and to the mass $\mathrm{M}$. We have noticed that deflection angle is gradually increasing for small variation of $M$. 
2. It is also noted that the deflection angle is gradually increasing for huge variation of $a$.

\section{Deflection angle w.r.t Deformation parameter:}

1. Here, it is is noticed that deflection angle gradually increasing for small variation of $M$.

2. It is also noticed that that deflection angle is exponentially increasing for the huge variation of $b$ for $4<b<8$.

\section{Deflection angle w.r.t M:}

1. It is observed that deflection angle is exponentially increasing for small variation of impact parameter and deflection angle rapidly increase for $0.1<b<0.2$.

2. It is also examined that deflection angle is gradually increasing for large variation of deformation parameter $a$.

In the future, the astrophysical observations might shed light on the effect of deformation parameter on the deflection angle. Any discovery of the deformation parameter would be an important signal beyond the general relativity.

[1] A. Einstein, Annalen Phys. 49, no. 7, 769 (1916) [Annalen Phys. 14, 517 (2005)].

[2] B. P. Abbott et al. [LIGO Scientific and VIRGO Collaborations], Phys. Rev. Lett. 116, no. 24, 241102 (2016).

[3] B. P. Abbott et al. [LIGO Scientific and VIRGO Collaborations], Phys. Rev. Lett. 118, 221101 (2017).

[4] K. Akiyama et al. [Event Horizon Telescope], Astrophys. J. Lett. 875, L1 (2019).

[5] D. I. Kazakov and S. N. Solodukhin, Nucl. Phys. B 429, 153-176 (1994).

[6] R. A. Konoplya, Phys. Lett. B 804, 135363 (2020)

[7] J. Peng, M. Guo and X. H. Feng, [arXiv:2008.00657 [gr-qc]].

[8] V. B. Bezerra, I. P. Lobo, J. P. Morais Graça and L. C. N. Santos, Eur. Phys. J. C 79, no.11, 949 (2019)

[9] T. Johannsen, C. Wang, A. E. Broderick, S. S. Doeleman, V. L. Fish, A. Loeb and D. Psaltis, Phys. Rev. Lett. 117, no. 9, 091101(2016).

[10] F. W. Dyson, A. S. Eddington and C. Davidson, Phil. Trans. Roy. Soc. Lond. A 220, 291-333 (1920).

[11] J. Soldner, Berliner Astronomisches Jahrbuch, 161-172. (1801).

[12] H. Hoekstra, M. Bartelmann, H. Dahle, H. Israel, M. Limousin, and M. Meneghetti, Space Sci. Rev. 177, 75 (2013).

[13] M. M. Brouwer et al., Mon. Not. R. Astron. Soc. 481, 5189 (2018).

[14] F. Bellagamba et al., Mon. Not. R. Astron. Soc. 484, 1598 (2019).

[15] N. Tsukamoto, T. Harada, and K. Yajima, Phys. Rev. D 86, 104062 (2012).

[16] N. Tsukamoto and T. Harada, Phys. Rev. D 87, 024024 (2013).

[17] N. Tsukamoto and Y. Gong, Phys. Rev. D 97, 084051 (2018).

[18] A. Abdujabbarov, B. Ahmedov, N. Dadhich and F. Atamurotov, Phys. Rev. D 96, no.8, 084017 (2017)

[19] H. Chakrabarty, A. B. Abdikamalov, A. A. Abdujabbarov and C. Bambi, Phys. Rev. D 98, no.2, 024022

[20] F. Atamurotov and B. Ahmedov, Phys. Rev. D 92, 084005 (2015)

[21] K.S. Virbhadra and G. F. R. Ellis, Phys. Rev. D 62, 084003 (2000).

[22] K. S. Virbhadra and G. F. R. Ellis, Phys. Rev. D 65, 103004 (2002)

[23] K. S. Virbhadra and C. R. Keeton, Phys. Rev. D 77, 124014 (2008)

[24] K. S. Virbhadra, Phys. Rev. D 79, 083004 (2009)

[25] C. R. Keeton, C. S. Kochanek and E. E. Falco, Astrophys. J. 509, 561-578 (1998).

[26] V. Bozza, Phys. Rev. D 66, 103001 (2002).

[27] V. Bozza, Gen. Rel. Grav. 42, 2269-2300 (2010).

[28] S. b. Chen and J. 1. Jing, Phys. Rev. D 80, 024036 (2009).

[29] M. Sharif and S. Iftikhar, Astrophys. Space Sci. 357, no.1, 85 (2015).

[30] W. G. Cao and Y. Xie, Eur. Phys. J. C 78, no.3, 191 (2018).

[31] G. S. Bisnovatyi-Kogan and O. Y. Tsupko, Universe 3, no.3, 57 (2017).

[32] G. S. Bisnovatyi-Kogan and O. Y. Tsupko, Mon. Not. Roy. Astron. Soc. 404, 1790-1800 (2010).

[33] P. V. P. Cunha and C. A. R. Herdeiro, Gen. Rel. Grav. 50, no.4, 42 (2018).

[34] R. M. Wald (1984), The University of Chicago Press 1984, 299-300.

[35] V. Bozza, and G. Scarpetta, Phys. Rev. D 76, 083008 (2007).

[36] P.C.W. Davies (1978), Reports on Progress in Physics 41 (8):1313. R. Astron. Soc. 2017, 468, 2605-2613.

[37] M. Bartelmann and P. Schneider, Phys. Rept. 340, 291 (2001).

[38] M. Sharif and S. Iftikhar, Astrophys. Space Sci. 357, no. 1, 85 (2015).

[39] G. W. Gibbons and M. C. Werner, Class. Quant. Grav. 25, 235009 (2008).

[40] M. C. Werner, Gen. Rel. Grav. 44, 3047-3057 (2012).

[41] K. Jusufi, M. C. Werner, A. Banerjee, and A. Ovgun, Phys. Rev. D 95, 104012 (2017).

[42] G. Crisnejo and E. Gallo, Phys. Rev. D 97, no.12, 124016 (2018).

[43] A. Ishihara, Y. Suzuki, T. Ono, T. Kitamura and H. Asada, Phys. Rev. D 94, no.8, 084015 (2016) 
[44] K. Jusufi, I. Sakalli, and A. Övgün, Phys. Rev. D 96, no.2, 024040 (2017).

[45] A. Övgün, K. Jusufi, and I. Sakalli, Annals Phys. 399, 193-203 (2018).

[46] K. Jusufi and A. Övgün, Phys. Rev. D 97, no.6, 064030 (2018).

[47] K. Jusufi, M. C. Werner, A. Banerjee, and A. Övgün, Phys. Rev. D 95, no.10, 104012 (2017).

[48] A. Övgün, Phys. Rev. D 99, no.10, 104075 (2019).

[49] W. Javed, R. Babar, and A. Övgün, Phys. Rev. D 100, no.10, 104032 (2019).

[50] W. Javed, J. Abbas, A. Övgün, Eur. Phys. J. C 79, no.8, 694 (2019).

[51] I. Sakalli and A. Övgün, EPL 118, no.6, 60006 (2017).

[52] K. de Leon and I. Vega, Phys. Rev. D 99, no.12, 124007 (2019).

[53] Z. Li and T. Zhou, Phys. Rev. D 101, no.4, 044043 (2020).

[54] A. Övgün, I. Sakalli, and J. Saavedra, Annals Phys. 411, 167978 (2019).

[55] A. Övgün, I. Sakalli, and J. Saavedra, JCAP 10, 041 (2018).

[56] K. Jusufi and A. Övgün, Int. J. Geom. Meth. Mod. Phys. 16, no. 08, 1950116 (2019).

[57] Q. M. Fu, L. Zhao and Y. X. Liu, [arXiv:2101.08409 [gr-qc]].

[58] Y. Kumaran and A. Övgün, Chin. Phys. C 44, no.2, 025101 (2020).

[59] R. Kumar, S. G. Ghosh and A. Wang, Phys. Rev. D 101, no.10, 104001 (2020).

[60] W. Javed, J. Abbas and A. Övgün, Phys. Rev. D 100, no.4, 044052 (2019).

[61] W. Javed, A. Hazma and A. Övgün, Phys. Rev. D 101, no.10, 103521 (2020).

[62] A. Ishihara, Y. Suzuki, T. Ono and H. Asada, Phys. Rev. D 95, no.4, 044017 (2017).

[63] T. Ono, A. Ishihara and H. Asada, Phys. Rev. D 96, no.10, 104037 (2017).

[64] T. Ono, A. Ishihara and H. Asada, Phys. Rev. D 99, no.12, 124030 (2019).

[65] T. Ono and H. Asada, Universe 5, no.11, 218 (2019).

[66] H. Arakida, Gen. Rel. Grav. 50, no.5, 48 (2018).

[67] Z. Li and A. Övgün, Phys. Rev. D 101, no.2, 024040 (2020).

[68] Z. Li, G. Zhang and A. Övgün, Phys. Rev. D 101, no.12, 124058 (2020).

[69] W. Javed, M. B. Khadim, A. Övgün and J. Abbas, Eur. Phys. J. Plus 135, no.3, 314 (2020).

[70] W. Javed, M. B. Khadim and A. Övgün, Eur. Phys. J. Plus 135, 595 (2020).

[71] Z. Li and J. Jia, Eur. Phys. J. C 80, no.2, 157 (2020).

[72] Z. Li and T. Zhou, [arXiv:2001.01642 [gr-qc]].

[73] K. Takizawa, T. Ono and H. Asada, Phys. Rev. D 101, no.10, 104032 (2020).

[74] G. W. Gibbons, Class. Quant. Grav. 33, no.2, 025004 (2016).

[75] S. U. Islam, R. Kumar and S. G. Ghosh, JCAP 09, 030 (2020).

[76] R. C. Pantig and E. T. Rodulfo, Chin. J. Phys. 66, 691-702 (2020).

[77] N. Tsukamoto, Phys. Rev. D 101, no.10, 104021 (2020).

[78] K. Jusufi, A. Övgün, and A. Banerjee, Phys. Rev. D 96, no.8, 084036 (2017).

[79] A. Övgün, Turk. J. Phys. 44, 465-471 (2020).

[80] A. Övgün and İ. Sakalli, Class. Quant. Grav. 37, no.22, 225003 (2020).

[81] A. Belhaj, M. Benali, A. E. Balali, H. El Moumni and S. E. Ennadifi, Class. Quant. Grav. 37, no.21, 215004 (2020).

[82] K. Jusufi, A. Övgün, J. Saavedra, Y. Vasquez, and P. A. Gonzalez, Phys. Rev. D 97, no.12, 124024 (2018).

[83] A. Övgün, K. Jusufi, and I. Sakalli, Phys. Rev. D 99, no.2, 024042 (2019).

[84] W. Javed, R. Babar, and A. Övgün, Phys. Rev. D 99, no.8, 084012 (2019).

[85] K. Jusufi and A. Övgün, Phys. Rev. D 97, no.2, 024042 (2018).

[86] K. Jusufi, A. Övgün, A. Banerjee and I. Sakalli, Eur. Phys. J. Plus 134, no.9, 428 (2019).

[87] A. Övgün, G. Gyulchev, and K. Jusufi, Annals Phys. 406, 152-172 (2019).

[88] A. Övgün, Universe 5, 115 (2019).

[89] A. Övgün, Phys. Rev. D 98, no.4, 044033 (2018).

[90] Z. Li, G. He and T. Zhou, Phys. Rev. D 101, no.4, 044001 (2020).

[91] T. Ono, A. Ishihara and H. Asada, Phys. Rev. D 98, no.4, 044047 (2018). 